\title{
Development of an FIA system with amperometric detection for determination of bentazone in estuarine waters
}

\author{
Rodrigo P. A. G. Cerejeira - Cristina Delerue-Matos \\ M. Carmo V. F. Vaz
}

\begin{abstract}
On the basis of its electrochemical behaviour a new flowinjection analysis (FIA) method with ampero- metric detection has been developed for quantification of the herbicide bentazone (BTZ) in estuarine waters. Standard solutions and samples $(200 \mu \mathrm{L})$ were injected into a water carrier stream and both $\mathrm{pH}$ and ionic strength were automatically adjusted inside the manifold. Optimization of critical FIA conditions indicated that the best analytical results were obtained at an oxidation potential of $1.10 \mathrm{~V}$, $\mathrm{pH} 4.5$, and an overall flow-rate of $2.4 \mathrm{~mL} \mathrm{~min}^{-1}$. Analysis of real samples was performed by means of calibration curves over the concentration range $2.5 \times 10^{-6}$ to $5.0 \times 10^{-5} \mathrm{~mol} \mathrm{~L}^{-1}$, and results were compared with those obtained by use of an independent method (HPLC). The accuracy of the amperometric determinations was ascertained; errors relative to the comparison method were below $4 \%$ and sampling rates were approximately 100 samples $\mathrm{h}^{-1}$. The repeatability of the proposed method was calculated by assessing the relative standard deviation (\%) of ten consecutive determinations of one sample; the value obtained was $2.1 \%$.
\end{abstract}

\section{Keywords}

Amperometry $\cdot$ Bentazone $\cdot$ FIA system $\cdot$ Herbicides . Estuarine waters

\section{Introduction}

Acidic herbicides have been identified in natural waters as a consequence of wide usage over the past ten years in several countries of the European Community. Among these herbicides, bentazone (BTZ, 3-isopropyl-1H-2,1,3benzothiadiazin-4-(3H)-one-2,2-dioxide) is commonly used in rice culture and is, as a consequence, frequently found in estuarine waters [1]. Analytical methods suitable for accurate determination of BTZ, not only in this kind of matrix but also in natural waters and in commercial products containing this herbicide, are therefore necessary.

Most methods reported in the literature are chromatographic techniques, particularly gas $[2,3,4,5,6]$ and liquid chromatography $[1,7,8,9,10,11,12]$. Determinations of BTZ using these conditions are time-consuming, because samples require previous physical treatment before assay; these procedures are, therefore, not considered satisfactory for routine determinations.

An electrochemical method [13] without these drawbacks has been developed for estimation of BTZ; it employs a voltammetric detection technique based on the electrochemical properties of the analyte. A severe limitation of this method is, however, the formation of reaction products that are strongly adsorbed on the glassy carbon electrode surface. This means that frequent cleaning of the electrode is necessary; this is unsuitable for routine determinations and might lead to irreproducible analysis. The method can, furthermore, only be used for the analysis of samples containing relatively large amounts of BTZ, because of its high detection limit $\left(10^{-5} \mathrm{~mol} \mathrm{~L}^{-1}\right)$. It is, therefore, inadequate for the quantification of BTZ in contaminated waters and, in particular, estuarine waters in which levels of herbicide are usually lower.

With the aim of eliminating these disadvantages of the voltammetric technique, a new analytical system is proposed. The need for high sensitivity, and thus low detection limits, linked with the electroanalytical oxidation of BTZ, suggested the selection of amperometric detection. With the purpose of enabling the analysis of a large number of samples per hour, the detector was coupled to continuous-flow analysis. Of the several flow analysis techniques described to date, the simplest and least expensive was selected - flow injection analysis (FIA).

\section{Experimental}

Apparatus

Solutions were pumped through the FIA equipment by means of a Gilson Minipuls 3 peristaltic pump, with pump tubing of the same 
brand. Standard solutions and samples were injected into the flow by means of a Rheodyne 5020 four-way rotary valve. Teflon $0.8 \mathrm{~mm}$ internal diameter tubing and connectors were used to link all the components of the FIA system. Mixing of two solutions inside the manifold was guaranteed by means of a home-made confluence, built as described elsewhere [14].

Amperometric detection was performed by use of a Metrohm 641 VA-detector and Metrohm 656 wall-jet cell. The working and reference electrodes were glassy carbon (Metrohm 6.0805.01) and $\mathrm{Ag} / \mathrm{AgCl} / \mathrm{KCl} 3.00 \mathrm{~mol} \mathrm{~L}^{-1}$ (Metrohm 6.0727.000), respectively. The auxiliary electrode was a gold electrode (Metrohm 6.530.320). Analytical signals from the detector were continuously monitored by means of a Kipp and Zonnen BD 111 recorder.

When necessary the glassy carbon electrode was mechanically cleaned by polishing its surface by use of a special kit (Metrohm $6.2802 .010)$, firstly with $\alpha-\mathrm{Al}_{2} \mathrm{O}_{3}(0.3 \mu \mathrm{m})$ until a shining surface was obtained and then with water. The electrode was then washed thoroughly with water and dried on tissue paper.

HPLC determinations [15] were performed with a Sykan A 1210 liquid chromatograph equipped with a model 3200 UV detector tuned to $225 \mathrm{~nm}$. Separation of sample components was accomplished on a Supercosil LC-18 column $(250 \mathrm{~mm} \times 4.6 \mathrm{~mm}, 5 \mu \mathrm{m}$ particle size $)$ from Macherey-Nagel, Germany.

$\mathrm{pH}$ was measured by means of a Metrohm $632 \mathrm{pH}$ meter connected to a combined glass electrode (Metrohm 6.020.000).

\section{Reagents and solutions}

BTZ (99.9\%) was purchased from Riedel-de Haën and used without further purification. All reagents were of analytical grade or equivalent and used without further purification. Deionized water (conductivity $<0.1 \mu \mathrm{S} \mathrm{cm}^{-1}$ ) was used throughout.

Britton-Robinson and acetate buffers of $\mathrm{pH}$ from 3.4 to 5.9 were tested as supporting electrolytes. The former were prepared by mixing different volumes of $0.16 \mathrm{~mol} \mathrm{~L}^{-1}$ phosphoric, acetic and boric acid standard solutions, then addition of $0.8 \mathrm{~mol} \mathrm{~L}^{-1}$ sodium hydroxide solution until the desired $\mathrm{pH}$ value was obtained [16]. The ionic-strength of the final solution was adjusted by addition of $1.34 \mathrm{~mol} \mathrm{~L}^{-1}$ potassium nitrate solution. Acetate buffer solutions were prepared by mixing different volumes of acetic acid and so-

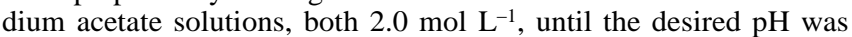
reached. Subsequent dilution was performed to furnish a final ionic strength of $0.2 \mathrm{~mol} \mathrm{~L}^{-1}$.

In the comparison method (chromatographic determination) solvents were of HPLC grade. Before use they were filtered and any dissolved air was removed with helium.

\section{Standard and sample preparation}

A stock standard solution of BTZ, concentration of $1.0 \times 10^{-4} \mathrm{~mol} \mathrm{~L}^{-1}$, was prepared by careful weighing of the solid and dissolution in water. The resulting solution was kept in the dark at $-4{ }^{\circ} \mathrm{C}$. Less concentrated solutions, with concentrations from $2.5 \times 10^{-6}$ to $5.0 \times 10^{-5} \mathrm{~mol} \mathrm{~L}^{-1}$, were prepared daily by dilution of the stock BTZ solution with water. These solutions were stable for at least one working day when kept in the dark (thus preventing decomposition of BTZ in the light).

Real samples of estuarine water were collected close to rice cultures located around Figueira da Foz, Portugal, 30 days after application of a commercial preparation containing BTZ. They were transported to the laboratory in dark flasks, at low temperature, and stored undisturbed under refrigeration for $24 \mathrm{~h}$, after which they were analysed by the two methods, without pretreatment, by use of the calibration-curve method. FIA analysis of the samples was performed directly whereas in HPLC the method of standard additions was used to fit the BTZ concentration in the samples within the concentration range of the calibration curve $-1.00 \mathrm{~mL}$ of the sample was added to $1.00 \mathrm{~mL}$ of a $5.0 \times 10^{-4} \mathrm{~mol} \mathrm{~L}^{-1} \mathrm{BTZ}$ standard.

\section{Comparison method}

Results from amperometric analysis of samples were compared with those obtained by use of an independent method employed by Riedel-de Haën for quality control of the pro analisi grade reagent [15]. HPLC was performed at room temperature with a mixture of water $(39.96 \%)$, acetonitrile $(60 \%)$, and phosphoric acid $(0.04 \%)$ as mobile phase at a flow rate of $1.4 \mathrm{~mL} \mathrm{~min}^{-1}$. Calibration was performed by injection of $20 \mu \mathrm{L}$ of BTZ standard solutions, with concentrations in the range $1.4 \times 10^{-4}$ to $7.1 \times 10^{-4} \mathrm{~mol} \mathrm{~L}^{-1}$, prepared in mobile phase.

\section{Results and discussion}

The main purpose of the FIA procedure used for BTZ determination was the direct use of samples by the system, thus eliminating any sample pretreatment. Any adjustment of the samples to conform with the requirements of the detector would be made inside the manifold.

The established FIA system had a double-channel (Fig. 1), which enabled the ionic strength and $\mathrm{pH}$ adjustments required by the detector. Standard solutions and samples were injected into a water carrier and combined with the supporting electrolyte at the confluence (X, Fig. 1), before reaching the detector. The effects of critical conditions were evaluated by a univariant process, i.e. varying

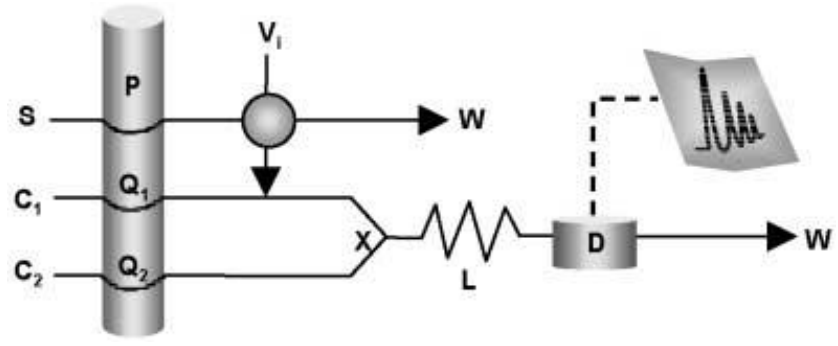

Fig. 1 Flow-injection system: $P$, peristaltic pump; $S$, sample; $C_{I}$, carrier stream (water); $C_{2}$, supporting electrolyte; $X$, confluence point; $Q_{1}=Q_{2}=1.2 \mathrm{~mL} \mathrm{~min}{ }^{-1} ; L$, reactor $(30 \mathrm{~cm}) ; V_{i}$, injection volume $(200 \mu \mathrm{L}) ; D$, detector; $W$, waste

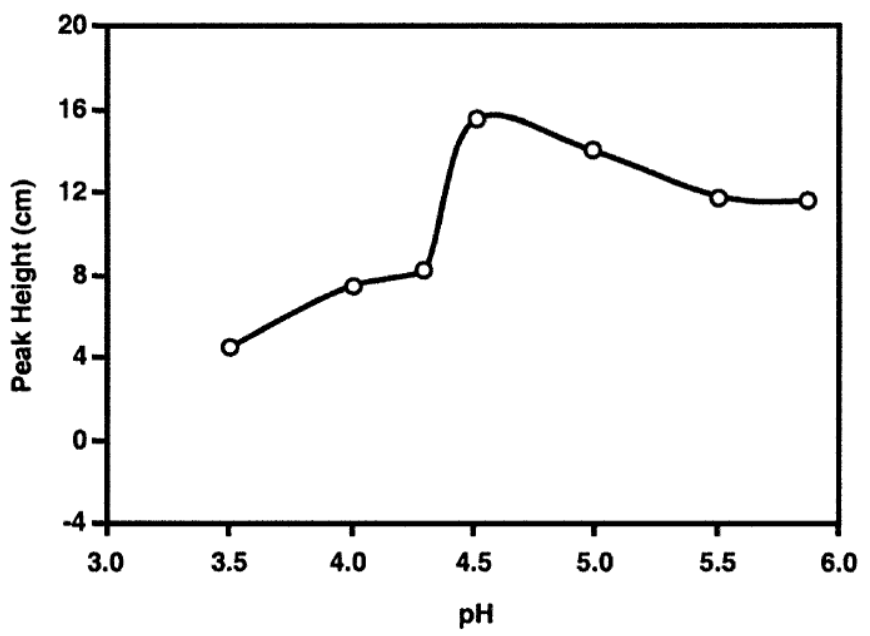

Fig. 2 Peak height $(\mathrm{cm})$ obtained for $5 \times 10^{-5} \mathrm{~mol} \mathrm{~L}^{-1}$ BTZ solution as function of the $\mathrm{pH}$ of the acetate buffer 
one condition each time and keeping the others constant. In this study, type and $\mathrm{pH}$ of the carrier electrolyte, the working electrode potential, flow-rates $\left(\mathrm{Q}_{1}\right.$ and $\mathrm{Q}_{2}$, Fig. 1), injection volume $\left(\mathrm{V}_{\mathrm{i}}\right.$, Fig. 1), and reactor length (L, Fig. 1) were tested. Optimization of these conditions was performed by analysis of $5.0 \times 10^{-5} \mathrm{~mol} \mathrm{~L}^{-1} \mathrm{BTZ}$ standard solution; experiments were designed to obtain the best balance of sensitivity, reproducibility, sampling rate, and economy of reagent.

Britton-Robinson and acetate buffers were tested as supporting electrolytes; the latter resulted in more sensitive response. Within the $\mathrm{pH}$ range considered the best results - higher absolute response and better reproducibility - were obtained at $\mathrm{pH} 4.5$ (Fig. 2).

With $\mathrm{pH} 4.5$ acetate buffer as the supporting electrolyte, the oxidation potential of the working electrode was varied from 0.60 to $1.30 \mathrm{~V}$ relative to $\mathrm{Ag} / \mathrm{AgCl}$ (Fig. 3). At potentials below $0.80 \mathrm{~V}$ no analytical signal was recorded. Increasing the potential led to a significant increase in peak height until $1.10 \mathrm{~V}$ was reached. The analytical signal was then fairly constant between 1.10 and $1.20 \mathrm{~V}$, beyond which the analytical response decreased significantly and reproducibility suffered accordingly. This behaviour of the detection system indicated that the range 1.10-1.20 V would lead to the highest possible sensitivity. To reduce possible interference from compounds other than BTZ present in real samples, the potential of $1.10 \mathrm{~V}$ was chosen.

Selection of the most appropriate flow rate was limited by the wall-jet cell; the manufacturer had advised against the use of high flow rates [17]. It was noted that overall flow rates $\left(Q_{1}+Q_{2}\right.$, Fig. 1) higher than $2.4 \mathrm{~mL} \mathrm{~min}{ }^{-1}$ were unsatisfactory because they gave rise to poor reproducibility. This was most probably because of high pressures within the system, owing to the mechanical characteristics of the electrochemical detector, the dead volume of which was approximately $1 \mu \mathrm{L}$. Although reducing the flow rate

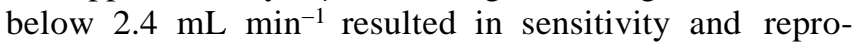
ducibility similar to those obtained at $2.4 \mathrm{~mL} \mathrm{~min}^{-1}$, the increased sampling rate at the higher flow led to the

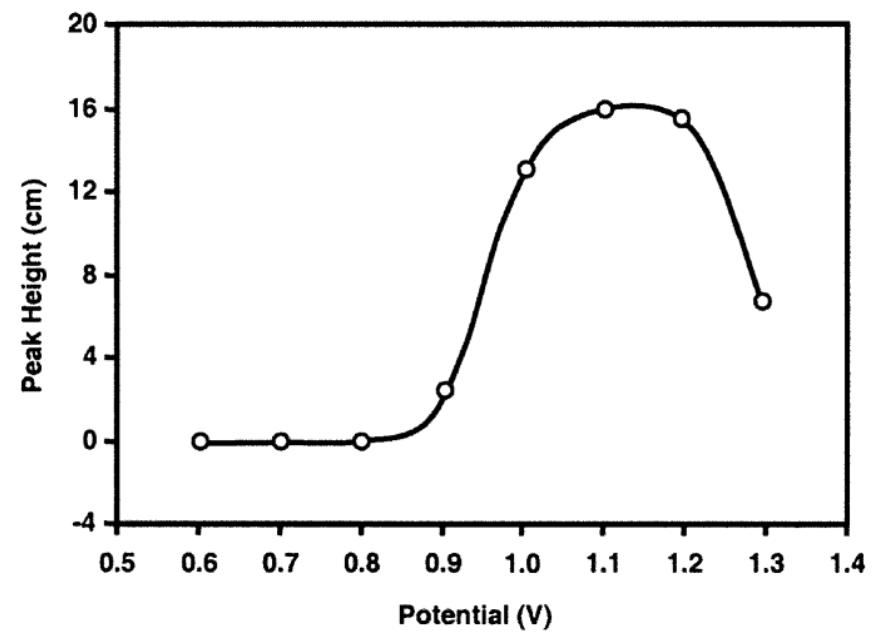

Fig. 3 Peak height $(\mathrm{cm})$ obtained for $5 \times 10^{-5} \mathrm{~mol} \mathrm{~L}^{-1}$ BTZ solution as function of the oxidation potential of the working electrode selection of $2.4 \mathrm{~mL} \mathrm{~min}{ }^{-1}$ for use in all further experiments.

The injection volume $\left(\mathrm{V}_{\mathrm{i}}\right.$, Fig. 1) was evaluated between 75 and $250 \mu \mathrm{L}$. Each volume injected into the manifold was ascertained by acid-base titration of the total volume collected after ten consecutive injections [18]. A volume of $200 \mu \mathrm{L}$ was selected as a compromise between high sensitivity, economy of reagents, and sampling rates. Different reactor lengths (L, Fig. 1) after the conflu- ence of sample, water, and supporting electrolyte streams were

tested. Increasing the length to $30 \mathrm{~cm}$ led to greater reproducibility and more efficient mixing of both streams. Above this value peak heights started decreasing, because of increasing dispersion of the analytical sample. For this reason, a $30 \mathrm{~cm}$ coil was selected.

In previous voltammetric determination of BTZ Triton-X 100 was used both to reduce adsorption of oxidation products at the glassy carbon surface and for stabilize the baseline [13]. Although the amount of mechanical cleaning required by the proposed method is quite low, the effect of Triton-X 100 was explored. Standard BTZ solutions containing different concentrations of Triton-X 100 were injected into a water stream and automatically mixed with supporting electrolyte carrying an equal concentration of Triton-X 100. No improvements in reproducibility were observed, suggesting the complete absence of the strong absorption of oxidation products reported in literature [13], most probably because of the low concentrations of BTZ and the short residence time of samples at the surface of the working electrode.

The determination limit, calculated under the optimized conditions and according to IUPAC recommendations [19], was $1.0 \times 10^{-6} \mathrm{~mol} \mathrm{~L}^{-1}$. Calibration curves were linear within the range $2.5 \times 10^{-6}$ to $5.0 \times 10^{-5} \mathrm{~mol} \mathrm{~L}^{-1} \mathrm{BTZ}$, with $R^{2}>0.998$.

\section{Interferences}

The samples used in this work could include pesticides other than BTZ. According to the source of application of the commercial BTZ product in rice cultures, they could also contain diquat, deltamethrin, and MCPA. Because levels of BTZ in estuarine waters were expected to be quite low, interference of these pesticides in the final analysis might be significant. Interference from diquat and deltamethrin was, therefore, evaluated by injecting pure solutions of each pesticide into the system under the optimized experimental conditions. No peak was recorded for either, indicating complete non-interference from both diquat and deltamethrin. Interference from MCPA was not evaluated because its oxidation potential is much higher than that of BTZ, eliminating the possibility of interference [20].

Application to real samples

After previous calibration four different samples were injected directly into the FIA system and amperometric 
Table 1 Results obtained from determinations of BTZ concentrations in estuarine waters by use of the FIA system and the comparison HPLC method, showing relative errors

\begin{tabular}{lrrr}
$\begin{array}{l}\text { Samples } \\
\left(\times 10^{-6} \mathrm{~mol} \mathrm{~L}^{-1}\right)\end{array}$ & \multicolumn{1}{l}{$\begin{array}{l}\text { FIA } \\
\left(\times 10^{-6} \mathrm{~mol} \mathrm{~L}\right.\end{array}$} & HPLC & ER $(\%)$ \\
\hline 1 & $4.70 \pm 0.19$ & $4.68 \pm 0.26$ & +0.3 \\
2 & $7.94 \pm 0.35$ & $8.22 \pm 0.24$ & -3.4 \\
3 & $14.0 \pm 0.53$ & $13.6 \pm 0.48$ & +2.9 \\
4 & $6.20 \pm 0.15$ & $6.10 \pm 0.27$ & +1.6 \\
\hline
\end{tabular}

quantification of BTZ was performed. The average values recorded after three trials for each sample are indicated in Table 1.

The precision of the results was confirmed by the small standard deviation of the mean results. Repeatability, calculated by determining the relative standard deviation for ten consecutive injections of a real sample, was found to be $2.1 \%$.

The accuracy of the results was confirmed by comparison with the results from HPLC determination; relative errors were always $<4 \%$. Because this independent method required addition of BTZ standard to the sample, which could lead to inaccuracy, the samples analysed were submitted to recovery trials. The recorded values ranged from $97.3 \%$ to $101.9 \%$, confirming the accuracy of amperometric analysis. The Student $t$-test was performed and the theoretical value was higher than that calculated for a 95\% confidence level, thus confirming the null hypothesis - that results from the two methods are in agreement [21].

Under the experimental conditions selected the system could be used to analyse approximately 100 samples $\mathrm{h}^{-1}$.

\section{Conclusions}

The FIA method developed here is simple, inexpensive, and enables the analysis of a large number of samples per hour without pretreatment. This method also has environmental advantages - it is considerably less polluting than liquid chromatography.

Compared with other electrochemical methods reported in the literature, this FIA amperometric method has both a low detection limit and a wide linear concentration range, and eliminates previous adsorption problems at the glassy carbon surface, which led to the need for frequent cleaning and increased the time per determination and reduced the reliability of the results.

These advantages enable recommendation of the method for routine analysis. This FIA method is an excellent alternative to conventional, chromatographic, or voltammetric, methods for determination of BTZ for contamination levels $>1.0 \times 10^{-6} \mathrm{~mol} \mathrm{~L}^{-1}$.

Acknowledgements The authors acknowledge Fundação para a Ciência e Tecnologia by the financial support (POCTI/35287/ AGR/1999).

\section{References}

1. Chiron S, Martinez E, Barceló D (1994) J Chromatogr 665: 283-293

2. Vink M, Vanderpoll JM (1996) J Chromatogr 733:361-366

3. David MD, Campbell S, Li QX (2000) Anal Chem 72:36653670

4. Kadokaami K, Morimoto M, Haraguchi K, Koja M, Schinohara R (1991) Anal Sci 7:247-252

5. Akerblom M, Alex G (1984) J Assoc Anal Chem 67:653-655

6. Miles CJ, Moye HA (1988) Anal Chem 60:220-226

7. Jeannot R, Sabik KH, Sauvard E, Genin E (2000) J Chromatogr 879:51-71

8. Hogenboom AC, Jagt I, Vreuls RJJ, Brinkman UAT (1997) Analyst 122:1371-1377

9. Balinova A (1996) J Chromatogr 728:319-324

10. Tanaka S, Ogata M (1995) Bunseki Kagaku 44:1067-1070

11. Liu CH, Mattern GC, Yu X, Rosen RT, Rosen JD (1991) J Agric Food Chem 39:718-721

12. Miles CJ, Doerge DR, Bajic S (1992) Arch Environ Contam Toxicol 22:247-250

13. Garrido EM, Lima JLFC, Delerue-Matos CM, Brett AMO (1998) Talanta 46:1131-1135

14. Alegret S, Alonso J, Bartroli J, Machado AASC, Lima JLFC, Paulis JM (1987) Quim Anal 6:278-285

15. Riedel-de Haën (1999) Certificate of analysis bentazon

16. Fernandes CM, Martin VC (1977) Talanta 24:747-748

17. Metrohm AG, CH-9100 Herisau (1984) Electrochemical detection in HPLC

18. Lima JLFC, Rangel AOSS (1990) J Int Sci Vigne Vin 24:4961

19. Miller JN (1991) Analyst 116:3-10

20. Garrido EM, Lima JLFC, Delerue-Matos CM, Brett AMO (1999) Portugaliae Electrochim Acta:17:313-317

21. McCormick D, Roach A (1987) Measurement statistics and computation. Wiley, Chichester 